\title{
A Fast and Robust Method for Image Segmentation Using Fuzzy Solutions of Partial Differential Equations
}

\author{
Yiliang Zeng ${ }^{1}$, Jinhui Lan ${ }^{1, *}$, Jinlin Zou ${ }^{1}$, Chunhong $\mathrm{Wu}^{1}$ and Juanjuan $\mathrm{Li}^{2}$ \\ ${ }^{1}$ School of Automation and Electrical Engineering, University of Science and \\ Technology Beijing, Beijing 100083, P.R. China \\ ${ }^{2}$ Beijing Wanji Techonology Co., Ltd, Haidian District, Beijing 100085, P.R. \\ China \\ ustbzengyiliang@163.com,ustblanjinhui@gmail.com
}

\begin{abstract}
In this paper, an efficient method for image segmentation with the help of the fuzzy solutions of partial differential equations is presented. We first designed a Poisson equation model for image segmentation using fuzzy solution technology, which aims to find a fuzzy solution to satisfy precisely the PDEs. Then, an appropriate segmentation model was obtained to extract the boundary of objects, according to the numerical characteristic in fuzzy solving process. Comparison with the previous approaches is provides to validate the validity of the proposed method. Experimental results on synthetic and real-world images demonstrate that the proposed method has good performances in terms of speed, accuracy, robustness against Gaussian noise, and effectiveness in shadow.
\end{abstract}

Keywords: Fuzzy solutions, image segmentation, Poisson equation, numerical characteristic

\section{Introduction}

In computer vision, image segmentation is a major process which aims to partition a given image into several regions with particular properties or to extract object of interest from the background [1-2]. This process is more challenging and critical in image analysis. It is well known that a lot of nature and physical phenomena can be modeled by Partial Differential Equations (PDEs). Hence, segmentation methods based on PDEs have attracted much attention and have became one of the main topics of modern image analysis. There are two main and basis approaches have been developed: the geodesic active contours method [3] and the Mumford and Shah approach [4]. The key idea that started the segmentation method based on PDEs fanfare was the active contour methods (ACMs), i.e., the 'snake' model, which achieves the goal of image segmentation by solving the minimum of energy functional. This was first done in the seminal work of Kass, Witkin and Terzopoulos [3]. However, it is a difficult task to choose a set of marker points for discretizing the parametrized evolving curve, and we could obtain different solutions by changing the parameters while preserving the same initial curve $[1,5-6]$. In order to overcome the above difficulties, the geodesic active contours method was proposed by Caselles, Kimmel, and Sapiro [7]. One of the interests in this method is that it can be rewritten using a level set formulation [1]. The Mumford and Shah approach is to find a close image of the initial one compounded of several regions with nearly constant intensity [1, 25-26].

How to find a numerical solution is one of the image segmentation problems based on PDEs as important as how to establish PDE model. However, the exact solutions to the PDEs cannot be calculated except for some simple and classical cases. In recent years, many methods have been developed for improving the performance of PDEs. Finite 
difference methods are famous and widely adopted in image segmentation field to solve some specific PDEs [8-9]. Generally, a finite difference solution can only offer one discrete solution. In addition, the average intensities inside and outside the contour should be computed at each iteration. This process increases drastically the CPU time by increasing communications between processors [2]. Level set methods [22] are also wellaccepted technology for solving PDEs. However, the conventional level set methods fall into some problems, such as processing in noisy images, producing accurate segmentation without prior information on the number of image segments and so on [23-26]. Hence, finding an effective and faster method to solve the PDEs for image segmentation is an important task. For this purpose, we propose a new method which tries to overcome the aforementioned drawbacks. Our method is based on a new idea which aims to find a suitable and fuzzy solution with adaptive algorithm for the PDEs.

Recently, the fuzzy theory has been used as an alternative approach for solving PDE. The fuzzy wavelets were demonstrated that it can be used for the solution of differential equations [10]. An approach with help of the fuzzy transform was used to numerical solutions of partial differential equations [11]. This method needs to solve a PDE with an algebraic equation through a fuzzy transform technique [12]. An advanced methodology based on fuzzy logic system and adaptive algorithm was successfully adopted to solve the PDE problem [13]. These methods can better handle the problem of time consuming and the algorithm is simple. In this paper, the fuzzy solutions of Partial Differential Equations are used to solve the image segmentation problem. The proposed method is based on the approach of PDE fuzzy model defined in [13]. The method is fast, robust against noise, and can detect objects efficiently.

The rest of this paper is organized as follows. In Section 2, a poisson equation model for image segmentation is designed. In Section 3, we explain the formulation of the proposed method. Section 4 validates the proposed method through experimental results. Section 5 concludes this paper.

\section{Poisson Equation Model for Image Segmentation}

In this section, we detail image segmentation model using poisson equation. Only the scalar image functions are considered, because the segmentation problem for each color component can be solved separately.

Figure 1 illustrates the notations: let $S$, a 2-D image, be the image definition domain, and let $o$ be the object with boundary $\partial \Omega$. Let $\mathrm{f}$ be the scalar function defined over $S$ minus the interior of $o$.

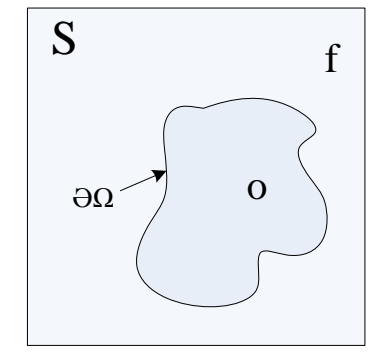

Figure 1. Minimization Problem Notations

The simple segmentation functions of $o$ from $S$ can be defined as the solution of the minimization problem [16] with Boundary Conditions (BCs),

$$
g(|\nabla S|)=\frac{1}{1+|\nabla S|^{2}} \text { with }\left.\mathbf{f}\right|_{\partial \Omega}=\left.o\right|_{\partial \Omega}
$$


Where $\nabla .=\left[\frac{\partial}{\partial x}, \frac{\partial}{\partial y}\right]$ is the gradient operator. The minimizer must satisfy the equation

$$
g(|\nabla S|)=0 \text { over } \Omega, \text { with }\left.\mathbf{f}\right|_{\partial \Omega}=\left.o\right|_{\partial \Omega}
$$

Where ${ }_{\Delta .}=\frac{\partial^{2}}{\partial x^{2}}+\frac{\partial^{2}}{\partial y^{2}}$ is the Laplacian operator. We also define a guidance field $\mathbf{v}=(u, v)$ which might be or not the gradient field of the object $o$. Then the minimization problem (1) can be extended as

$$
g\left(|\nabla S-\mathbf{v}|^{2}\right)=\frac{1}{1+|\nabla S-\mathbf{v}|^{2}} \text { with }\left.\mathbf{f}\right|_{\partial \Omega}=\left.o\right|_{\partial \Omega}
$$

Its solution is the unique solution of the following Poisson equation with Dirichlet boundary conditions [15],

$$
\Delta S=\operatorname{div} \mathbf{v} \text { over } \Omega \text {, with }\left.\mathbf{f}\right|_{\partial \Omega}=\left.o\right|_{\partial \Omega}
$$

Where $\operatorname{div} \mathbf{v}=\frac{\partial u}{\partial x}+\frac{\partial v}{\partial y}$ is the divergence of $\mathbf{v}$.

The Poisson equation model for image segmentation (4) can be rewritten as the following form

$$
\frac{\partial^{2} S}{\partial x^{2}}+\frac{\partial^{2} S}{\partial y^{2}}=\frac{\partial u}{\partial x}+\frac{\partial v}{\partial y} \text { with }\left.\mathbf{f}\right|_{\partial \Omega}=\left.o\right|_{\partial \Omega}
$$

\section{Our Segmentation Method}

The unknown solution of the Poisson equation for image segmentation with the BCs in (5) can be regarded as an unknown system, and it can be discretized and solved in a number of ways. And the fuzzy logic system is very suitable for treating this problem with its excellent approximation ability [18].

\subsection{Parameters and Symbols Description}

For images, the underlying pixel grid can be naturally used as the discrete grid. For each pixel $p$ of $S$, let $N_{p}$ be the set of its connected neighbors. The size of $S$ is $M \times N$, and $S(x, y)$ represents the pixel value.

In this paper, we cut the image into regions with $m \times n$ piexls. $a_{i}^{x}$ and $a_{j}^{y}$ are the mean values of a row or column in $S(i, j)$. Then we also define the initial boundary for (5) in $S(i, j)$ as follows, the left side of boundary $B_{l e f t}=\bigcup_{y=1}^{n} S_{i j}(1, y)=H(1, y)$, the right side $B_{\text {right }}=\bigcup_{y=1}^{n} S_{i j}(m, y)=H(m, y)$, the up side $B_{u p}=\bigcup_{x=1}^{m} S_{i j}(x, n)=H(x, n)$, and the down side $B_{\text {down }}=\bigcup_{x=1}^{m} S_{i j}(x, 1)=H(x, 1)$. Figure 2 is shown to explain some parameters clearly. 


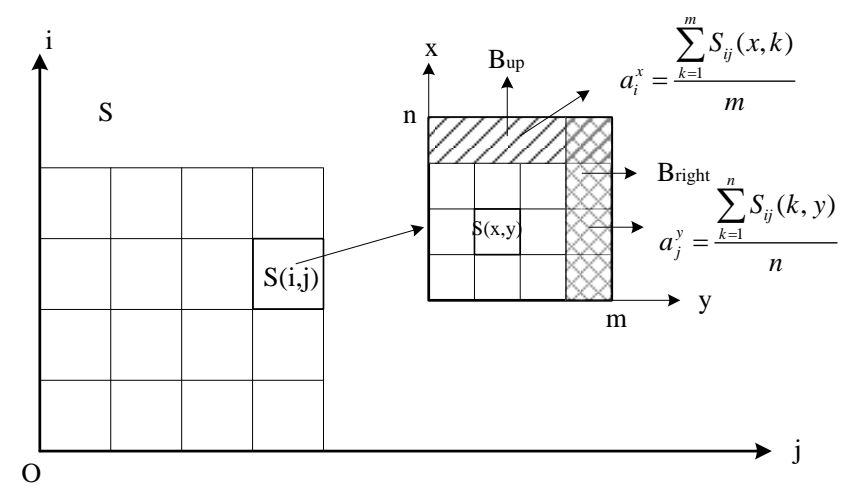

Figure 2. Illustration for Same Parameters

\subsection{Algorithm Design Description}

The next sections detail the steps to processing each region separately based on the design procedure, which was proposed by Yung-Yue Chen, $\mathrm{Yu}-\mathrm{Te} \mathrm{Chang}$, and Bor-Sen Chen in [13].

\section{(1) Construct fuzzy rule bases}

With the help of fuzzy solutions design procedure of Partial Differential Equations, the Poisson equation with the BCs in (5) can be described as follow:

$$
\begin{gathered}
\mathfrak{R}^{\alpha}: I F x \text { is } \mu_{i}^{x} \text { and } y \text { is } \mu_{j}^{y} \\
\text { THEN } \phi(x, y)=\theta_{i j}
\end{gathered}
$$

where $\mathfrak{R}^{\alpha}(\alpha=1, \ldots, m \times n)$ are fuzzy rules, and $x$ and $y$ are the input variables to the fuzzy system. $\mu_{i}^{x}(i=1, \ldots, m)$ and $\mu_{j}^{y}(j=1, \ldots, n)$ are the membership functions that characterize the $i$ th and $j$ th fuzzy sets defined in the space of the variables of $x$ and $y$ coordinates, respectively. $\phi(x, y)=\theta_{i j}$ is the approximated fuzzy solution proposed by the $\alpha$ th rule.

In this paper, the membership functions are chosen as $\mu_{i}^{x}=\exp \left[-\frac{1}{2}\left(x-a_{i}^{x}\right)^{2}\right]$ and $\mu_{j}^{y}=\exp \left[-\frac{1}{2}\left(y-a_{j}^{y}\right)^{2}\right]$.

(2) Build the fuzzy solution function

The fuzzy basis functions are constructed as $\xi_{i j}=\frac{\mu_{i}^{x} \mu_{j}^{y}}{\sum_{k=1}^{m} \sum_{l=1}^{n} \mu_{i}^{k} \mu_{j}^{l}}$.

Collect the all the $\xi$ of each point in $S(i, j)$ into a vector $\underline{\xi_{i j}}=\left[\xi_{i j}^{11}, \xi_{i j}^{12}, \ldots, \xi_{i j}^{m n}\right]^{T}$. It can be figured out that $\sum_{k=1}^{m} \sum_{l=1}^{n} \xi_{i j}^{k l}=1$.

Make the parameters $\theta_{i j}$ initially assigned as $\theta_{i j}^{11}=\theta_{i j}^{12}=\ldots=\theta_{i j}^{m n}=0.5$. And collect them into vector

$$
\theta_{i j}=\left[\theta_{i j}^{11}, \theta_{i j}^{12}, \ldots, \theta_{i j}^{m n}\right]^{T}
$$

Then, the fuzzy solution is constructed as $\phi(x, y)=\underline{\theta_{i j}} \underline{\xi_{i j}}$. 
It means that $\underline{\theta_{i j}}$ is the interpolated by the fuzzy smooth functions $\underline{\xi_{i j}}$ to approximate the exact solution $\phi(x, y)$ of the PDE [13].

(3) Re-construct the Poisson equation

By substituting $\phi(x, y)$ into (5), then (5) becomes

$$
{\underline{\theta_{i j}}}^{T} F=\frac{\partial u}{\partial x}+\frac{\partial v}{\partial y}
$$

Where

$$
F=\left[\begin{array}{l}
\xi_{i j}^{11} \\
\xi_{i j}^{12} \\
\vdots \\
\xi_{i j}^{m n}
\end{array}\right]=\left[\begin{array}{l}
\xi_{i i}^{11}+\xi_{j j}^{11} \\
\xi_{i i}^{12}+\xi_{j j}^{12} \\
\vdots \\
\xi_{i i}^{m n}+\xi_{j j}^{m n}
\end{array}\right]
$$

and

$$
\xi_{i i}^{x y}=\frac{\left(\frac{\mu_{j}^{y}}{\sum_{l=1}^{n} \mu_{j}^{l}}\right)^{2} \partial\left(\frac{\mu_{i}^{x}}{\sum_{k=1}^{m} \mu_{i}^{k}}\right)^{2}}{\partial x^{2}}, \xi_{j j}^{x y}=\frac{\left(\frac{\mu_{i}^{x}}{\sum_{k=1}^{m} \mu_{i}^{k}}\right)^{2} \partial\left(\frac{\mu_{j}^{y}}{\sum_{l=1}^{n} \mu_{j}^{l}}\right)^{2}}{\partial y^{2}}
$$

We also define that $L(1, y)=\bigcup_{y=1}^{n} \xi_{i j}(1, y), L(m, y)=\bigcup_{y=1}^{n} \xi_{i j}(m, y), L(x, n)=\bigcup_{x=1}^{m} \xi_{i j}(x, n)$, and $L(x, 1)=\bigcup_{x=1}^{m} \xi_{i j}(x, 1)$.

Then the boundary conditions are changed into

$$
\begin{aligned}
& \theta_{i j}{ }^{T} L(1, y)=B_{\text {left }}=H(1, y) \\
& {\hat{\theta_{i j}}}^{T} L(m, y)=B_{\text {right }}=H(m, y) \\
& {\theta_{i j}}^{T} L(x, n)=B_{u p}=H(x, n) \\
& {\underline{\theta_{i j}}}^{T} L(x, 1)=B_{\text {down }}=H(x, 1)
\end{aligned}
$$

(4) Construct energy function

Five error functions can be defined for calculate the fuzzy solution for (5),

$$
\begin{aligned}
& \beta_{0 i j}\left(x, y, \underline{\theta_{i j}}\right)=\underline{\theta_{i j}}{ }^{T} F-\frac{\partial u}{\partial x}-\frac{\partial v}{\partial y} \\
& \beta_{1 i j}\left(y, \underline{\theta_{i j}}\right)=\underline{\theta_{i j}{ }^{T}} L(1, y)-H(1, y) \\
& \beta_{2 i j}\left(y, \underline{\theta_{i j}}\right)=\underline{\theta_{i j}}{ }^{T} L(m, y)-H(m, y) \\
& \beta_{3 i j}\left(x, \underline{\theta_{i j}}\right)={\overline{\theta_{i j}}}^{T} L(x, n)-H(x, n) \\
& \beta_{4 i j}\left(x, \underline{\theta_{i j}}\right)=\underline{\theta_{i j}} L(x, 1)-H(x, 1)
\end{aligned}
$$

And the energy function is constructed [13] as follows, 


$$
\begin{aligned}
E= & \left\{\sum_{x=1}^{m} \sum_{y=1}^{n} \frac{1}{2}\left[\beta_{0 i j}\left(x, y, \underline{\theta_{i j}}\right)\right]^{2}\right\}+\left\{\sum_{y=1}^{n} \frac{1}{2}\left[\kappa_{1} \beta_{1 i j}\left(y, \underline{\theta_{i j}}\right]^{2}\right\}\right. \\
& +\left\{\sum_{y=1}^{n} \frac{1}{2}\left[\kappa_{2} \beta_{2 i j}\left(y, \underline{\theta_{i j}}\right)\right]^{2}\right\}+\left\{\sum_{x=1}^{m} \frac{1}{2}\left[\kappa_{3} \beta_{3 i j}\left(x, \underline{\theta_{i j}}\right)\right]^{2}\right\} \\
& +\left\{\sum_{x=1}^{m} \frac{1}{2}\left[\kappa_{4} \beta_{4 i j}\left(x, \underline{\theta_{i j}}\right]^{2}\right\}\right. \\
= & \frac{1}{2} S_{i j}{ }^{T} S_{i j}
\end{aligned}
$$

Where

$$
\begin{aligned}
& \underline{S_{i j}}=\left[\beta_{0 i j}\left(1,1, \underline{\theta_{i j}}\right) \quad \beta_{0 i j}\left(1,2, \underline{\theta_{i j}}\right)\right. \\
& \left.\cdots \kappa_{1} \beta_{1 i j}\left(n, \theta_{i j}\right) \cdots \kappa_{4} \beta_{4 i j}\left(m, \underline{\theta_{i j}}\right)\right]^{T}
\end{aligned}
$$

And $\kappa_{1}=\kappa_{2}=\kappa_{3}=\kappa_{4}=\kappa$ are weighting factors. The number of matrix element is $(m n+2(m+n))$.

According to reference [13], the parameter vector $\underline{\theta_{i j}}$ can be calculated by the following formula,

$$
\frac{d \theta_{i j}}{d t}=-\eta_{\theta} \frac{\partial S_{i j}{ }^{T}}{\partial \underline{\theta_{i j}}} S_{i j}
$$

where $\eta_{\theta}$ is the adjustment parameter.

(5) Construct the solver model based on a numerical characteristic

In order to use the formula for image processing, we make Equation (12) simplified to

$$
\frac{d \theta_{i j}}{d t}=\eta_{\theta} \frac{\frac{\partial S_{i j}{ }^{T}}{\partial \theta_{i j}} / \partial t}{\partial t} S_{i j}
$$

Then (13) can be rewrote as

$$
\frac{d \theta_{i j}}{d t} \frac{\partial \theta_{i j}}{\partial t}=\eta_{\theta} \frac{\partial S_{i j}{ }^{T}}{\partial t} \underline{S_{i j}}
$$

Because the ${\underline{S_{i j}}}_{t}{ }^{T}=\frac{\partial S_{i j}{ }^{T}}{\partial t}$ can be discretized with its neighbor, and described as

$$
{\underline{S_{i j}}}_{t}{ }^{T}(k)=\underline{S_{i j}}{ }^{T}(k+1)-\underline{S_{i j}{ }^{T}}(k)
$$

where $k=1, \ldots,(m n+2(m+n))$.

Then a numerical characteristic of (14) can be figured out that, when processing the image areas which are in the flat site, the result of dot products between ${\underline{S_{i j}}}_{t}{ }^{T}(k)$ and $S_{i j}$ is very close to zero. And when processing the boundary areas, the result is far great than zero. Therefore, we can use this numerical characteristic to extract the edge information.

Finally, we define an alternative fuzzy solution, which avoids calculating the approximation by iteration, for the image segmentation problem of PDEs and BCs in equation (5) as follows,

$$
\left.\phi_{a \_i j}(x, y)=\mid \eta_{\theta} \underline{S_{i j}{ }^{T}} t \underline{\left[S_{i j}\right.}\right) \mid
$$

The physical meaning of this segmentation model can be organized as follows: it considers the relationship not only between adjacent pixels, but also between the 
pixel and the edge of processing region. And then the relationship is used to locate object contour with numerical characteristic.

\section{Experiments and Analysis}

This section is divided into two parts. The first part illustrates quantitative performance comparison and evaluation on synthetic images. The second part evaluates the performance of our method in real-world images experiments. We compared the proposed method with the well-known Chan-Vese (CV) method [5] and Distance Regularized Level Set Evolution (DRLSE) described in [8]. All the methods have been implemented using Matlab R2010b which is installed on a PC Core 2 Duo T6570 processor with a clock speed of $2.10 \mathrm{GHz}$ and $2 \mathrm{~GB}$ of RAM. In most of these experiments, we used weighting factors $\kappa=5$ and adjustment parameter $\eta_{\theta}=5$.

\subsection{Quantitative Performance Comparison and Evaluation on Synthetic Images}

To assess accuracy and running time, we applied the proposed method and comparative approaches to the synthetic images which included six different graphs with $600 \times 600$ pixels in figure 3. The first image of Fig.3 (a) shows the original input image, and the second, the third, and the fourth images are imposed with Gaussian white noise of mean $0.02,0.2$ and 0.5 , respectively. Fig. 3 (b) shows the segmentation result using the method by Chan et al, Fig.3 (c) shows the segmentation result by using the DRLSE method, and Fig.3 (d) shows the segmentation result by using the proposed method. In this experiment, we set iteration times $t \leq 1000$ for CV method and DRLSE method. The initial zero level contour was a circle with centre in the middle of the image and diameter equal to the height of the images in CV method, while the DRLSE method used two rectangles which were half of the image as initial zero level contour.

In order to objectively evaluate the proposed method, three widely used metrics, the Hausdorff Distance (HD) [21], Peak Signal to Noise Ratio (PSNR) and CPU running time were used. The lower HD is, the better the segmentation result is. And the higher PSNR is, the better the segmentation result is. The PSNR and Hausdorff Distance values of proposed method, CV and DRLSE for synthetic images with different Guassian Noise are shown in Fig.4. All the CPU times are displayed in Table 1.

Table 1. CPU Times of the Experiment Shown in Figure 3

\begin{tabular}{|c|c|c|c|c|}
\hline \multirow{2}{*}{ Methods } & \multicolumn{4}{|c|}{ CPU times (s) } \\
\cline { 2 - 5 } & $\begin{array}{c}\text { Original } \\
\text { image }\end{array}$ & $\begin{array}{c}\text { Image with Gaussian } \\
\text { Noise of mean 0.02 }\end{array}$ & $\begin{array}{c}\text { Image with Gaussian } \\
\text { Noise of mean 0. 2 }\end{array}$ & $\begin{array}{c}\text { Image with Gaussian } \\
\text { Noise of mean 0.5 }\end{array}$ \\
\hline CV & 261.756374 & 272.963299 & 278.227348 & 251.823734 \\
\hline DRLSE & 559.431114 & 566.835717 & 583.833102 & 563.176144 \\
\hline $\begin{array}{c}\text { Proposed } \\
\text { method }\end{array}$ & 26.866496 & 28.012624 & 27.372500 & 28.659734 \\
\hline
\end{tabular}

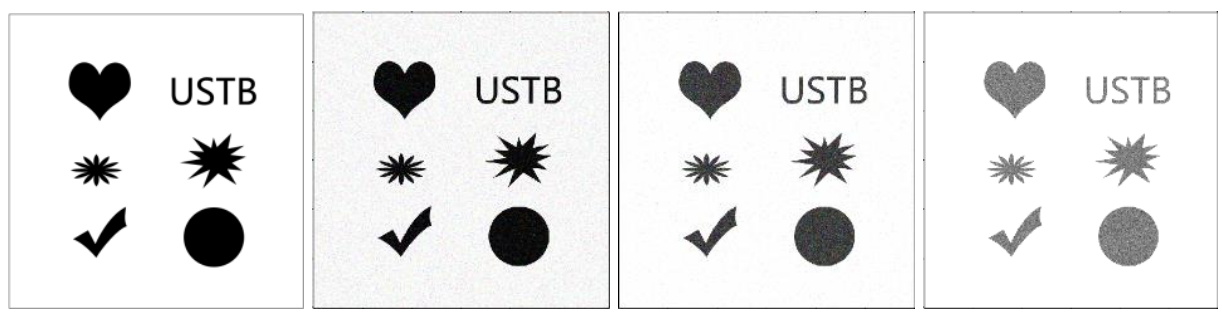

(a) 

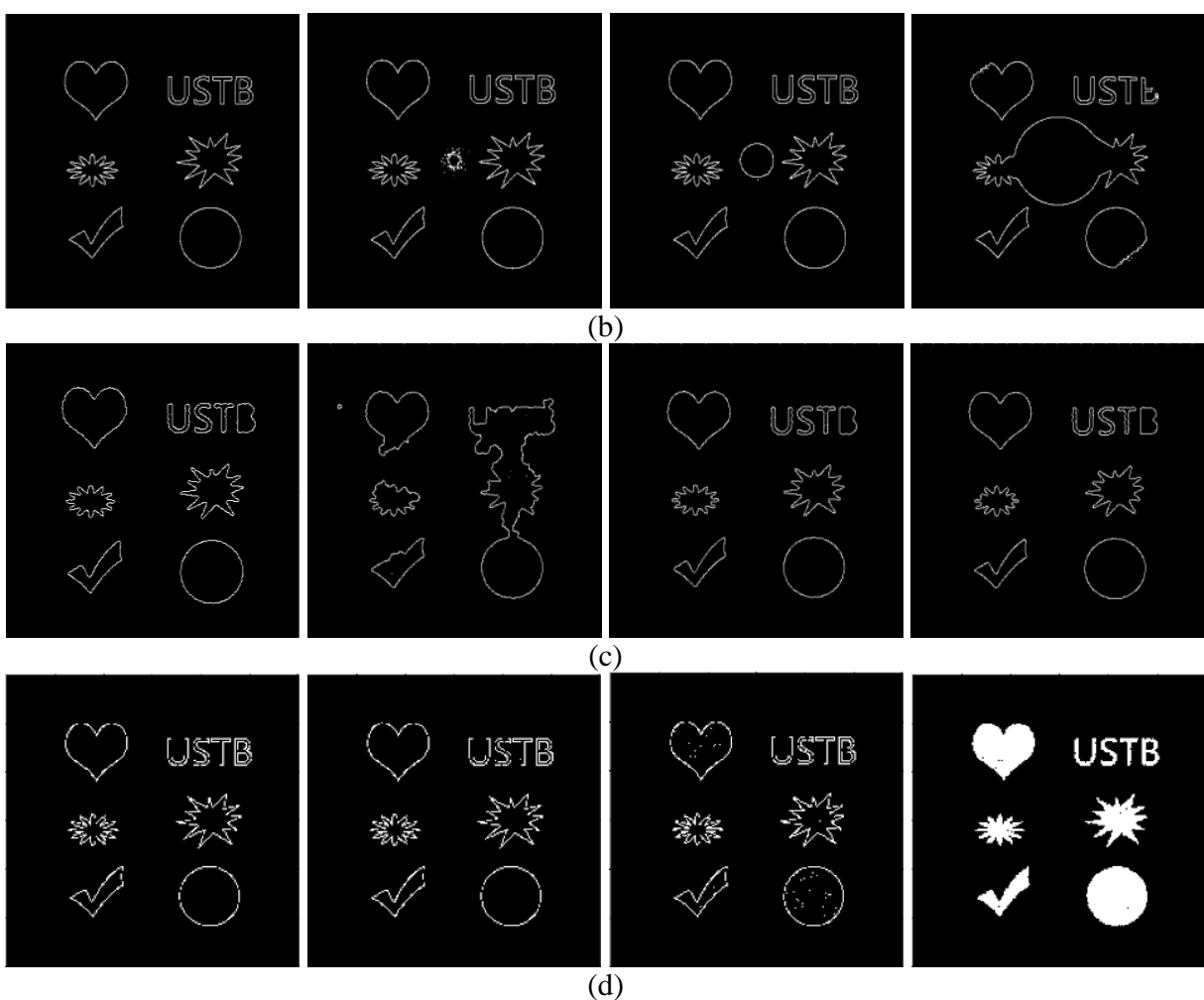

(d)
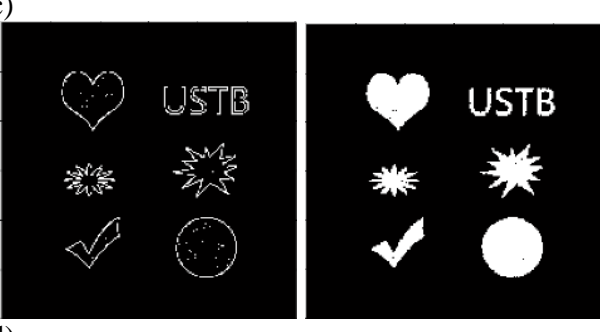

Figure. 3. Segmentation of Synthetic Images. (a) Original Input Images with Different Noise, (b) Segmentation Results of CV Method, (c) Segmentation Results of DRLSE Method, and (d) Segmentation Results of the Proposed Method

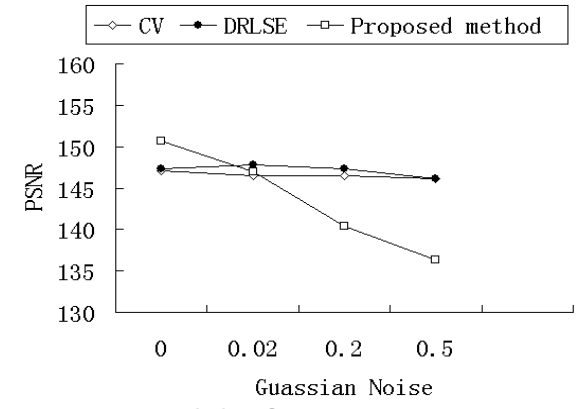

(a)PSNR values

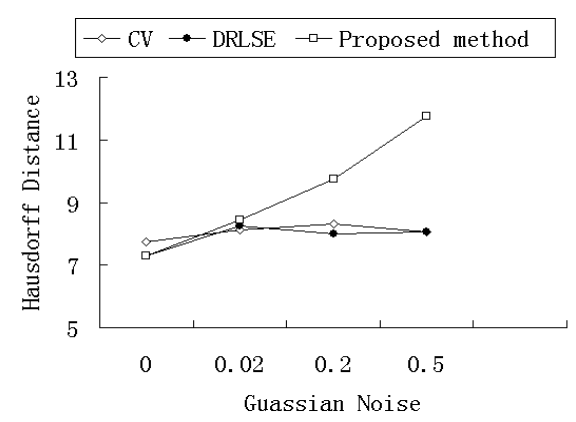

(b) Hausdorff Distance values

Figure 4. Performance Comparison of Proposed Method, CV and DRLSE for
Synthetic Images with Different Guassian Noise

The CV method could be good for edge information, and the PSNR and HD values were steady. However, the processing time of $\mathrm{CV}$ method increases with the increase of noise. The center of graph in Fig.3 (b) represents the final circle level curve for the 1000 iteration times. It can be seen clearly that there are still lots of areas have not been processed in the last image. In Fig.3 (c), the DRLSE method can extract the complete external contour for each graph in images. However, it could not detect the internal contour like ' $\mathrm{B}$ ' in the second graph. Also it could not achieve concave area completely. In other world, the DRLSE method could not achieve satisfactory effects for the similar graphs shown in second row.

Although our proposed method might cause discontinuity of contours in some place, the segmentation results can extract the image boundary information effectively in Fig.3 
(d). Despite the PSNR values becoming more and more small with the increase of noise, they are still in a quite high level. The range of PSNR are 136 150 (Fig.4), which is only the relative instability comparing to other methods. There is another advantage of our method that the Guassian Noise is only influential to the contour inside. Taking of the fourth image in Fig.3 (d) as an example, the HD values become higher due to the change of inner areas of objects. The outer regions of objects are still smooth like other images in Figure 3 (d).

In addition, we can see from Table.I that the proposed method gave the acceptable results in shortest time for the images with different Gaussian Noise. The average processing time of proposed method for these synthetic images was about 28 seconds as shown in Table I. But the average processing time of CV method and DRLSE method less than 1000 iterations were about 270 seconds and 570 seconds, respectively. They were nearly 10 and 20 times than our method.

\subsection{Experiments for Segmenting Real Images}

The proposed method has been applied to real-world images. In order to describe the performance of algorithms, we set the number of iterations $t$ for $\mathrm{CV}$ and DRLSE method not more than 1000 . We also made the mask with holes around the whole image as the initial zero level contours for $\mathrm{CV}$ method, and this code was implemented by Yue $\mathrm{Wu}$ [19], Tufts University. A rectangle initial zero level contour around the whole image was used for DRLSE method, and this code exists on the internet [20]. Fig.5 shows the segmentation results of the proposed method, $\mathrm{CV}$ method and DRLSE method for several real-world images.

Figure 5 (1)-(3) show the results for real images with different objects, an image of a pot, an image with four coins and an image of two spanners. The sizes of these images are $740 \times 500,240 \times 300$, and $240 \times 320$ pixels. In the example, all the contours of objects could be found by each method. However, the pot object and its shadow were not segmented completely by CV and DRLSE method in Figure 5 (1), while our method was able to find all the objects with good accuracy even under the background of a shadow.

Figure 5 (4) and (5) show the results for nature images which include different background in each image. Fig.5 (4) is a welcoming pine image with $320 \times 540$ pixels, and Fig. 5 (5) is a bird image with $480 \times 720$ pixels. The DRLSE method could segment the blue sky in Fig.5 (4) and the green background in Figure 5 (5). Also the external contour of objects was extracted accurately. In Fig.5 (4), our method is capable of segmentation of the same quality as CV method. But in Fig.5 (5b), the CV method using holes mask is powerless for the green background. Using our method, it is easy to eliminate the influence of background.

Figure 5 (6)-(8) shows the results for remote sensing images. Figure 5 (6) and (7) are satellite images with $360 \times 360$ and $420 \times 600$ pixels, respectively. Figure 5 (8) is an image of a galaxy with $220 \times 300$ pixels. Because the complexity of the objects, it was unable to complete the image segmentation in 1000 iterations by DRLSE method. Also some contours of small regions could not be found, such as the small island around the earth in Figure $5(7 \mathrm{c})$. The proposed method obtained the results similar to those by using the $\mathrm{CV}$ method.

Figure 5 (9)-(11) show the results for one object real images. Figure 5 (9) and (10) are images of the transportation, and Figure 5 (11) is an image of person. The sizes of these images are $768 \times 1024,480 \times 720$, and $660 \times 520$. It can be shown that the result of CV is sensitive to the shadows in Figure 5 (9b) and (10b). DRLSE can obtain good results in images of transportation (Figure 5 (9c) and (10c)), but it cannot get a correct segmentation result in Figure 5 (11c). Our method is perceptually more precise and complete than other methods at the object boundary. 
It can be seen from Figure 5, although our method is not continued in a small area of object boundary, it can still obtain the same quality of segmentation results as CV method. And the proposed method was less sensitive to shadows than CV. In order to further evaluate the performance of our method, the CPU time of real-world images in Figure 5 are plotted in Figure 6. As seen from Figure 6, the running time of $\mathrm{CV}$ method is dramatically varied according to different sizes of image and different complexity of image content. The CPU time under the processing of our method keeps a fast and robust running speed. On average, our CPU time is 20.3807 seconds and $\mathrm{CV}$ is 171.6952 seconds in processing the eleven images.

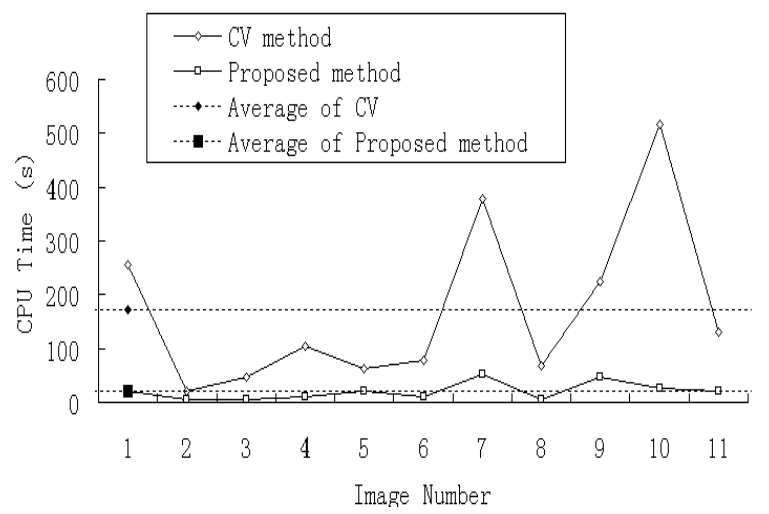

Figure 6. CPU Time of CV Method and Proposed Method for Real-world Images

\section{Conclusion}

In this paper, a new image segmentation method using fuzzy solutions of Partial Differential Equations (PDEs) is proposed. The Poisson Equation of image segmentation is solved by using the powerful and simple technology, which can obtain an alternative solution using numerical characteristic. Then, the proposed method uses the numerical characteristic to exact the boundary information of object by considering the neighbor of each pixel and its relationship to region boundary. Experimental results on synthetic and real-world images indicate that our method give as good result as classical method based on PDE. The good performance of the proposed method in terms of speed, accuracy, robustness against Gaussian noise, and effectiveness in shadow is also demonstrated in this paper. Future works can be an implementation of the proposed method with continuous boundary and against salt and pepper noise.

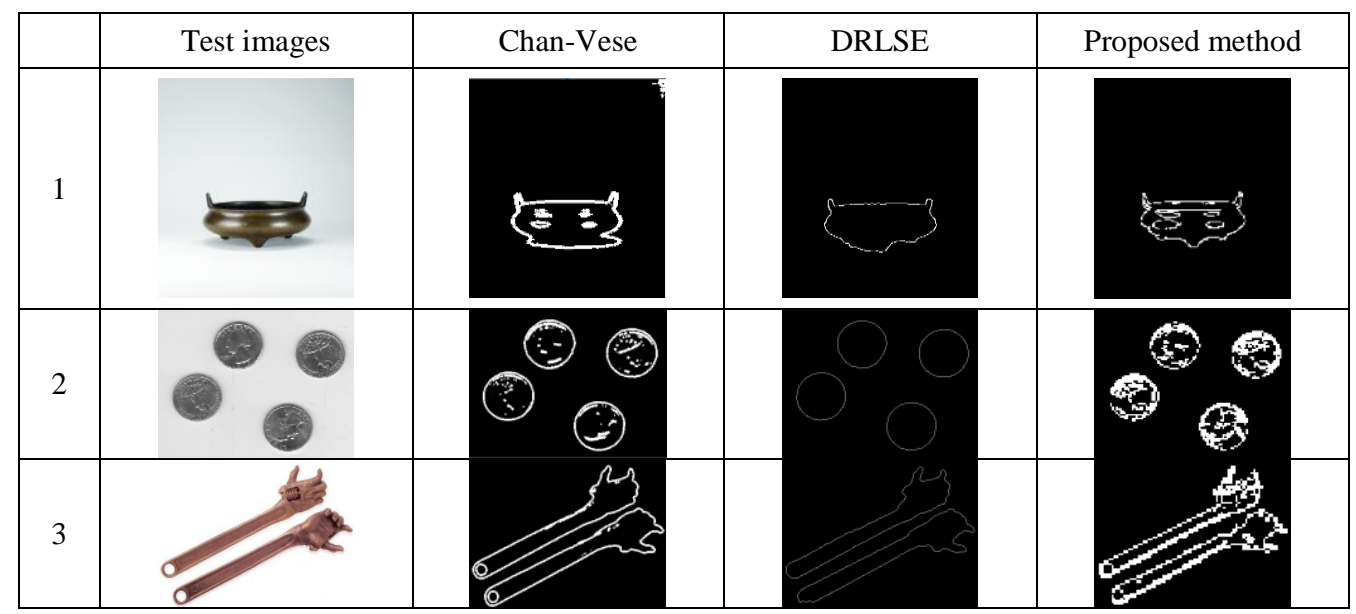




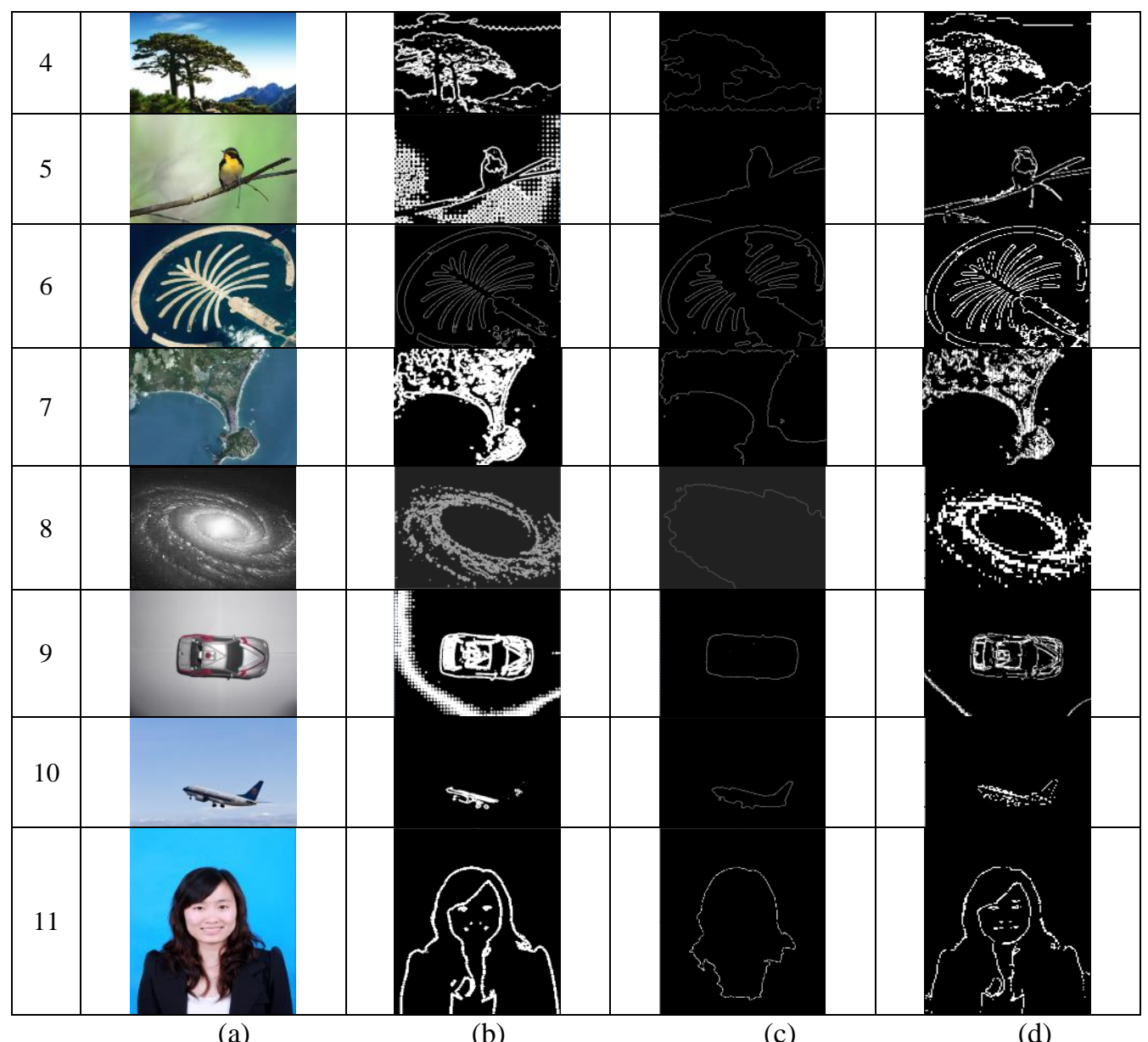

(a)

(b)

(c)

(d)

Figure 5. Segmentation of real-world images. (a) Original input images, (b) segmentation results of CV method, (c) segmentation results of DRLSE method, and (d) segmentation results of the proposed method.

\section{Acknowledgements}

This work was supported by the National Natural Science Foundation of China (Grant No. 61174181) and National High Technology Research and Development Program of China (863 Program, Grant No. 2012AA12A302-4).

\section{References}

[1] A. Gilles and P. Kornprobst, "Mathematical Problems in Image Processing: Partial Differential Equations and the Calculus of Variations", Springer, New York (2006).

[2] B. A. Souleymane, X. B. Gao and B. Wang, "A Fast and Robust Level Set Method for Image Segmentation Using Fuzzy Clustering and Lattice Boltzmann Method", IEEE Transactions on Cybernetics, vol. 43, no. 3, (2013).

[3] M. Kass, A. Witkin and D. Terzopoulos, "Snakes: active contour models", International Journal of Computer Vision, vol. 1, no. 4, (1988).

[4] D. Mumford and J. Shah, "Optimal Approximations by Piecewise Smooth Functions and Associated Variational Problems", Communications on Pure and Applied Mathematics, vol. 42, (1989).

[5] T. Chan and L. Vese, "Active contours without edges", IEEE Transactions on Image Processing, vol. 10, no. 2, (2001).

[6] C. Y. Xu and J. L. Prince, "Snakes, Shapes, Gradient Vector Flow", IEEE Transactions on Image Processing, vol. 7, no. 3, (1998).

[7] V. Caselles, R. Kimmel, and G. Sapiro, "Geodesic Active Contours", International Journal of Computer Vision, vol. 22, no. 1, (1997).

[8] C. M. Li, C. Y. Xu, C. F. Gui and M. D. Fox, "Distance Regularized Level Set Evolution and Its Application to Image Segmentation”, IEEE Transactions on Image Processing, vol. 19, no. 12, (2012). 
[9] R. M. Spitaleri, M. March, and D. Arena, "A Multigrid Finite-difference Method for the Solution of Euler Equations of the variational Image Segmentation”, Applied Numerical Mathematics, vol. 39, no. 2, (2001).

[10] A. Shmilovici and O. Maimon, "On the solution of differential equations with fuzzy spline wavelets", Fuzzy Sets and Systems, vol. 96, no. 1, (1998).

[11] M. Stepnicka and R. Valasek, "Numerical Solutio of Partial Differential Equations with Help of Fuzzy Transform", Proceedings of the IEEE International Conference on Fuzzy Systems, (2005), Reno, NV, USA, June.

[12] I. Perfilieva, "Fuzzy Transforms: Theory and Applications", Fuzzy Sets and Systems, vol. 157, no. 8, (2005).

[13] Y. Y. Chen, Y. T. Chang, and B. S. Chen, "Fuzzy Solutions to Partial Differential Equations: Adaptive Approach", IEEE Transactions on Fuzzy Systems, vol. 17, no. 1, (2009).

[14] S. P. Banks, "Control Systems Engineering: Modeling and Simulation, Control Theory", and Microprocessor Implementation. Upper Saddle River, NJ: Prentice-Hall, (1986).

[15] P. Patrick, G. Michel, and B. Andrew, "Poisson Image Editing", ACM Transactions on Graphics, vol. 22, no. 3, (2003).

[16] P. Perona and J. Malik, "Scale-Space and Edge Detection using Anisotropic Diffusion", IEEE Transactions on Pattern Analysis and Machine Intelligence, vol. 12, no. 7, (1990).

[17] Z. X. Yang, F. Lu, and Y. S. Li, "Computation of Image Gradient and Divergence and Their Application to Edge Detection of Noisy Images", ACTA Scientiarum Nuturalium Universitatis Sunyatseni, vol. 41, no. 6, (2002).

[18] E. Elbasi, "Fuzzy Logic-Based Scenario Recognition from Video Sequences", Journal of Applied Research and Technology, vol. 11, no. 5, (2013).

[19] Y. Wu, "Active Contour without Edge Matlab Code", http://sites.google.com/site/ rexstribeof- image processing/.

[20] C. M. Li, "DRLSE Matlab Code", http://www.imagecomputing.org/ cmli/.

[21] S. Chabrier, H. Laurent, C. Rosenberger, and B. Emile, "Comparative study of contour detection evaluation criteria based on dissimilarity measures", EURASIP Journal of Image and Video Processing, 2008, vol. 2, (2008).

[22] S. Osher and J. Sethian, "Fronts propagating with Curvature-Dependent Speed: Algorithms based on Hamilton-Jacobi formulations", Journal of Computational Physics, vol. 79, no. 1, (1988).

[23] C. Y. Li, X. Y. Wang, S. Eberl, M. Fulham and D. Feng, "A new energy framework with distribution descriptors for image segmentation", IEEE Transactions on Image Processing, vol. 22, no. 9, (2013).

[24] C. M. Li, C. Y. Kao, J. C. Gore, and Z. H. Ding, "Minimization of region-scalable fitting energy for image segmentation", IEEE Transactions on Image Processing, vol. 17, no. 10, (2008).

[25] L. A. Vese and T. F. Chan, "A multiphase level set framework for image segmentation using the mumford and shah model", International journal of computer vision, vol. 50, no. 3, (2002).

[26] J. Lie, M. Lysaker, and X. C. Tai, "A binary level set model and some application to Mumfordshah image segmentation”, IEEE Transactions on Image Processing, vol. 15, no. 5, (2006). 